\title{
A Novel Case of Severe Respiratory Symptoms and Persistent Pulmonary Hypertension in a Saudi Neonate With SARS-CoV-2 Infection
}

\author{
Kumail B. Algadeeb ${ }^{1}$, Haider H. AlMousa ${ }^{1}$, Sajjad M. AlKadhem ${ }^{2}$, Mohammed O. Alduhilan II ${ }^{1}$, Yameen \\ Almatawah $^{3}$
}

1. Neonatology, Maternity and Children Hospital, Al-Ahsa, SAU 2. Pediatrics, Maternity and Children Hospital, AlAhsa, SAU 3. Pediatric Infectious Disease, Maternity and Children Hospital, Al-Ahsa, SAU

Corresponding author: Kumail B. Algadeeb, kalgadeeb@moh.gov.sa

\begin{abstract}
Coronavirus disease 2019 (COVID-19) is an emergent disease that has spread rapidly to infect more than 210 countries across the world. With the increasing number of infected pregnant women, many physicians hypothesized the perinatal transmission as a potential route of transmission. Some cases of perinatal transmission have been described, but it is unclear if these occurred via the transplacental or the transcervical routes or through environmental exposure. In this report, we described a case of a female infant who was delivered by caesarean section at 34 weeks' gestation to an infected mother. The neonate was transferred into the Neonatal Intensive Care Unit (NICU) Level 3, with the precaution of airborne and contact isolation. All required investigations were performed, including blood gases, nasopharyngeal swab, chest $\mathrm{x}$-ray, and echocardiogram. On the fifth day of delivery, her investigations demonstrated a positive severe acute respiratory syndrome coronavirus 2 (SARS-CoV-2) infection. Despite applying all recommended guidelines and following the treatment protocol, she developed severe respiratory symptoms with persistent pulmonary hypertension, which progressed significantly to her death.
\end{abstract}

Received 09/02/2020

Review began 09/06/2020 Review ended 09/10/2020 Published 09/15/2020

\section{() Copyright 2020}

Algadeeb et al. This is an open access article distributed under the terms of the Creative Commons Attribution License CC-BY 4.0., which permits unrestricted use, distribution, and reproduction in any medium, provided the original author and source are credited.
Categories: Obstetrics/Gynecology, Pediatrics, Infectious Disease

Keywords: pregnancy, covid-19, sars-cov-2, neonate, case report, saudi arabia, pphn

\section{Introduction}

Coronavirus disease 2019 (COVID-19) has spread rapidly across the world since December 2019, when it initially emerged in Wuhan (Hubei, China), and millions of confirmed cases have been reported worldwide [1]. It has also been named severe acute respiratory syndrome coronavirus 2 (SARS-CoV-2). The number of pregnant women infected with the SARS-CoV-2 virus is increasing. SARS-COV-2 is mainly transmitted through droplets, but other routes have been hypothesized. Some cases of perinatal transmission have been described, but it is unclear if these occurred via the transplacental or the transcervical routes or through environmental exposure [2-4]. As far as we know, there have been only two reports with effective investigation proving the vertical transmission in neonates born to mothers with positive perinatal SARSCoV-2 $[5,6]$.

Since the emergence of the pandemic in March 2020, 45 pregnant women with positive reverse transcription polymerase chain reaction (RT-PCR) results for SARS-CoV-2 have been admitted into our institute, Maternity and Children's Hospital in Al-Ahsa, Saudi Arabia. All of their newborns were screened for SARSCoV-2 following the Ministry of Health COVID-19 protocol [7]. There were only three neonates with positive results for the test by nasopharyngeal RT-PCR. Notably, only one of these three neonates was symptomatic and required advanced intensive care management, and this is the case that we are reporting in this paper.

\section{Case Presentation}

A 37-year-old primigravida woman visited our obstetric emergency department on June 2020, at 31 weeks' gestation with intermittent bloody spotting and leg swelling. She had a history of pregnancy-induced hypertension on methyldopa treatment and gestational diabetes controlled through her diet. Fundal ultrasonography showed a low-lying placenta. The patient was admitted for bleeding observation and medical management. She completed a dexamethasone course six days after admission, and the course was not repeated.

One day before the delivery, the patient experienced shortness of breath and fever $\left(38^{\circ} \mathrm{C}\right)$. On examination, she demonstrated harsh bronchial breathing on chest auscultation, and she required low-flow oxygen to maintain her oxygen saturation above $90 \%$. Her chest x-ray showed bilateral multifocal peripheral airspace opacities, suggesting COVID-19 (Figure 1). 


\section{Cureus}

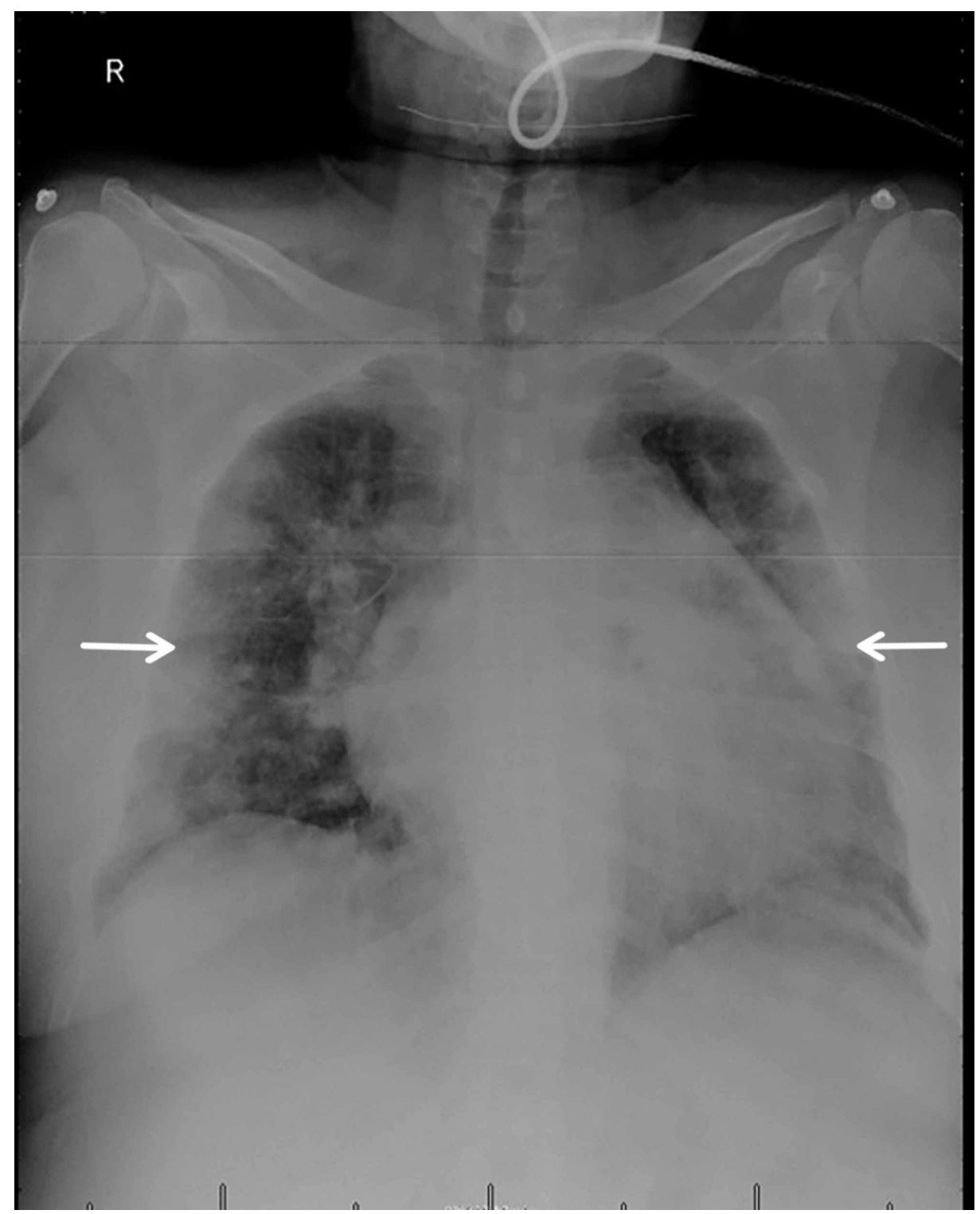

FIGURE 1: Chest $x$-ray of the mother with positive SARS-CoV-2 infection shows bilateral multifocal peripheral airspace opacities.

SARS-CoV-2, severe acute respiratory syndrome coronavirus 2

She was isolated immediately, and a nasopharyngeal swab for SARS-CoV-2 PCR testing was taken. A high vaginal swab and urine culture were negative for bacterial growth, and there were no signs of chorioamnionitis. Cardiotocography (CTG) was not reassuring, with a decrease in beat-to-beat variability; therefore, an emergency caesarean was planned.

On the day of delivery, a female infant was delivered by caesarean section at 34 weeks' gestation, with a birth weight of 2,475 $\mathrm{g}$ (on the 70th percentile), head circumference of $30 \mathrm{~cm}$ (on the 22nd percentile), and length of $43 \mathrm{~cm}$ (on the 25 th percentile). She cried immediately after birth, and the Apgar score was 8 and 8 at 1 and 5 minutes, respectively. The baby was intubated at 15 minutes of age, and positive pressure ventilation was provided because she was grunting, severely retracting, and having low oxygen saturation despite application of continuous positive airway pressure (CPAP) and a fraction of inspired oxygen $\left(\mathrm{FiO}_{2}\right)$ of $100 \%$. The baby shifted to the Neonatal Intensive Care Unit (NICU) Level 3, with the precaution of airborne and contact isolation. All staff who attended the delivery were following full precautions as per the protocol for suspected or confirmed COVID-19 cases.

In the NICU, the neonate was sedated and mechanically ventilated, requiring high ventilatory pressures and $\mathrm{FiO}_{2}$ of $100 \%$, with a differential saturation of more than 10 between pre- and post-ductal oxygen

saturations. Even with higher settings, it was difficult to ventilate and oxygenate the baby optimally, and 


\section{Cureus}

hence baby was shifted to high-frequency ventilation with a mean airway pressure of $17 \mathrm{~cm} \mathrm{H}_{2} \mathrm{O}$, a frequency of $15 \mathrm{~Hz}$, and an amplitude of $30 \mathrm{~cm} \mathrm{H}_{2} \mathrm{O}$. The first x-ray of the chest showed the ground-glass appearance of respiratory distress syndrome (Figure 2).

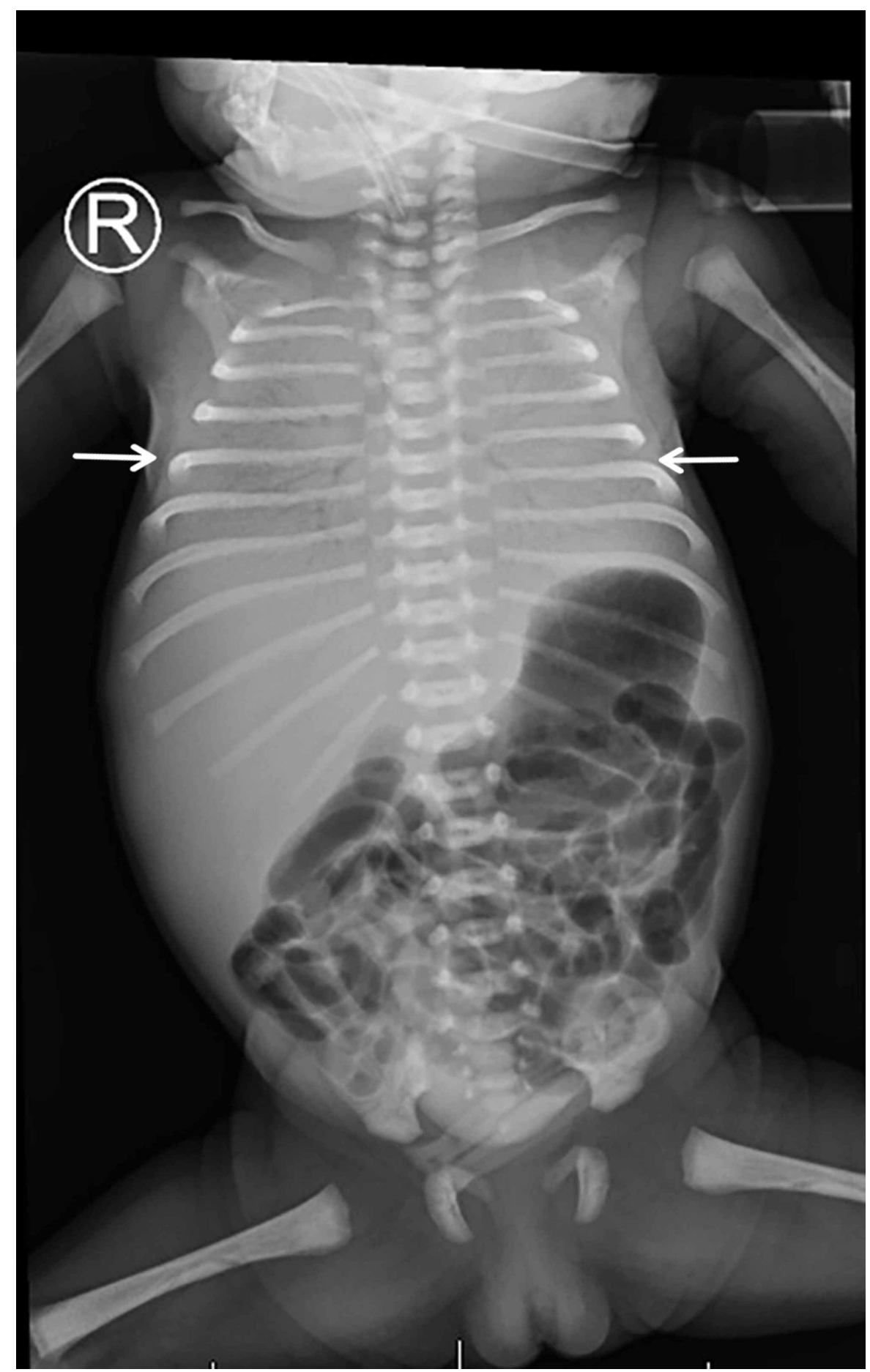

FIGURE 2: First chest $x$-ray of the neonate shows bilateral ground-glass appearance suggesting respiratory distress syndrome. An endotracheal tube was high in this $\mathrm{x}$-ray.

The baby received two doses of surfactant through the endotracheal tube, but there was no improvement in the ventilator setting or $\mathrm{FiO}_{2}$ requirement. Repeated chest x-rays showed bilateral perihilar hazy opacities with bronchial wall thickness, more evident on the left side, with mildly hyperinflated lungs (Figure 3). 


\section{Cureus}

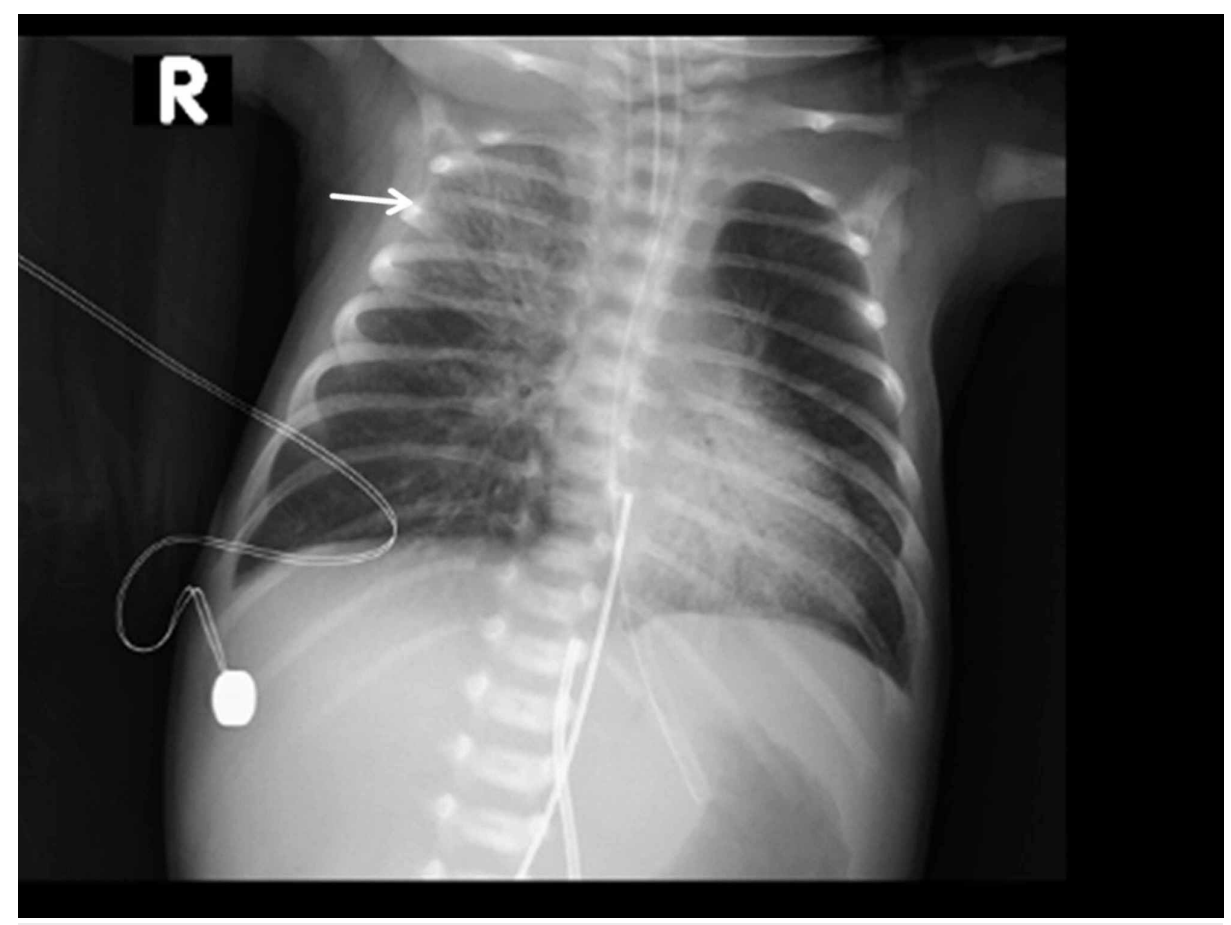

FIGURE 3: Chest $x$-ray of the neonate at day 2 of life shows bilateral perihilar hazy opacities with bronchial wall thickness, more evident at the left side with mildly hyperinflated lungs.

The neonate's blood gas showed an element of metabolic acidosis, with a $\mathrm{PO}_{2}$ of 77 and an oxygen index of 23. The electrocardiogram was normal, and no differences were noticed in four limb blood pressure readings. The echocardiogram indicated severe persistent pulmonary hypertension, severe tricuspid regurgitation, and moderate mitral regurgitation with an estimated pulmonary pressure of $76 \mathrm{mmHg}$, a bidirectional shunt seen at the level of the patent foramen ovale, and a right-to-left shunt through the large patent ductus arteriosus. There was a normal ventricular function with an ejection fraction of $66 \%$ and fractional shortening of $34 \%$. Therefore, the neonate started on inhaled nitric oxide at a dose of $20 \mathrm{ppm}$, and by the end of the first 24 hours of life, her circulation was being supported with $15 \mu \mathrm{g} / \mathrm{kg} / \mathrm{min}$ of dobutamine and $15 \mu \mathrm{g} / \mathrm{kg} / \mathrm{min}$ of dopamine to maintain her mean blood pressure above $40 \mathrm{mmHg}$.

The baby remained nil by mouth and started on full total parenteral nutrition with blood sugar and frequent laboratory monitoring (Table 1). The infectious disease team was involved, and sepsis workup was performed. The neonate was started on ampicillin and cefotaxime. Cultures of blood and cerebrospinal fluid showed negative for bacterial growth. C-reactive protein had a positive value of $6 \mathrm{mg} / \mathrm{L}$. 


\begin{tabular}{|c|c|c|c|c|c|}
\hline \multicolumn{3}{|c|}{ Laboratory result at first day } & \multicolumn{2}{|c|}{ Laboratory result at 24 hours prior death } & \multirow{2}{*}{$\begin{array}{l}\text { Reference range } \\
12.5-13.7 \mathrm{~g} / \mathrm{dL} \\
\end{array}$} \\
\hline \multirow{3}{*}{ Complete blood count } & $\mathrm{Hgb}(\mathrm{g} / \mathrm{dL})$ & 15 & $\mathrm{Hgb}(\mathrm{g} / \mathrm{dL})$ & 14 & \\
\hline & WBC $\left(\times 10^{3} / \mu \mathrm{L}\right)$ & 26 & WBC $\left(\times 10^{3} / \mu \mathrm{L}\right)$ & 23 & $4.5-13.5 \times 10^{3} / \mu \mathrm{L}$ \\
\hline & Platelets $\left(\times 10^{3} / \mu \mathrm{L}\right)$ & 70 & Platelets $\left(\times 10^{3} / \mu \mathrm{L}\right)$ & 64 & $150-350 \times 10^{3} / \mu \mathrm{L}$ \\
\hline \multirow{2}{*}{ Coagulation profile } & PTT (seconds) & 44 & PTT (seconds) & 43 & $30-40$ seconds \\
\hline & INR & 1 & INR & 1 & $0.8-1.1$ \\
\hline \multirow{4}{*}{ Electrolytes } & $\mathrm{Na}(\mathrm{mmol} / \mathrm{L})$ & 129 & $\mathrm{Na}(\mathrm{mmol} / \mathrm{L})$ & 152 & 135-145 mmol/L \\
\hline & $\mathrm{Ca}(\mathrm{mmol} / \mathrm{L})$ & 1.9 & $\mathrm{Ca}(\mathrm{mmol} / \mathrm{L})$ & 2.2 & $2.1-2.5 \mathrm{mmol} / \mathrm{L}$ \\
\hline & $\mathrm{K}(\mathrm{mmol} / \mathrm{L})$ & 5 & $\mathrm{~K}(\mathrm{mmol} / \mathrm{L})$ & 4.8 & $3.5-5 \mathrm{mmol} / \mathrm{L}$ \\
\hline & $\mathrm{PO}_{4}(\mathrm{mmol} / \mathrm{L})$ & 2 & $\mathrm{PO}_{4}(\mathrm{mmol} / \mathrm{L})$ & 1.6 & $0.78-1.42 \mathrm{mmol} / \mathrm{L}$ \\
\hline \multirow{2}{*}{ Renal profile } & $\operatorname{SrCr}(\mu \mathrm{mol} / \mathrm{L})$ & 98 & $\mathrm{SrCr}(\mu \mathrm{mol} / \mathrm{L})$ & 132 & $44-88 \mu \mathrm{mol} / \mathrm{L}$ \\
\hline & BUN (mmol/L) & 3 & BUN (mmol/L) & 11 & 1.7-7.1 mmol/L \\
\hline \multirow{3}{*}{ Liver profile } & ALT (unit/L) & 8.8 & ALT (unit/L) & 48 & $10-30$ unit/L \\
\hline & AST (unit/L) & 41 & AST (unit/L) & 23 & 13-35 unit/L \\
\hline & Total bilirubin (mg/dL) & 5.9 & Total bilirubin (mg/dL) & 19 & $0.2-1.5 \mathrm{mg} / \mathrm{dL}$ \\
\hline
\end{tabular}

\section{TABLE 1: Basic laboratory result for the neonate.}

Hgb: haemoglobin, WBC: white blood cell, PTT: partial thromboplastin time, INR: international normalized ratio, ALT: alanine aminotransferase, AST: aspartate transaminase, SrCr: serum creatinine, BUN: blood urea nitrogen.

At the third day of life, the mother was confirmed to be SARS-CoV-2 positive; therefore, nasopharyngeal and tracheal swabs for SARS-CoV-2 were taken from the neonate for PCR testing, which revealed a positive result by the fifth day of life. Our hospital follows the Supportive Care and Treatment Protocol of the Saudi Ministry of Health for Patients Confirmed with COVID-19, which recommends using hydrocortisone for preterm infants with a corrected gestation age of $<40$ weeks: (IV) $0.5 \mathrm{mg} / \mathrm{kg}$ every 12 hours for seven days and then $0.5 \mathrm{mg} / \mathrm{kg}$ once daily for three days [7]. This was the protocol followed in this case.

The creatinine level rose, accompanied by a decrement in the urine output, indicating acute kidney injury. There was a substantial drop in the platelet count during the hospital stay, and the baby required frequent platelet transfusions. She also required a blood transfusion once. The coagulation profile was normal. By day 6 , the neonate developed an abnormal cyclic movement in all limbs associated with decreasing oxygen saturation and tachycardia; therefore, she was loaded with phenobarbital followed by maintenance doses. A brain ultrasound showed grade III germinal matrix haemorrhage with mild dilatation in lateral ventricles.

Over the following days, the neonate remained critically ill with cardiorespiratory failure, persistent pulmonary hypertension, and acute kidney injury. Antibiotics were upgraded to imipenem/cilastatin and vancomycin. SARS-CoV-2 PCR testing was repeated twice 72 hours apart, and the results were positive in all samples. She was on high-frequency ventilation and nitric oxide, with low oxygen saturation and poor perfusion supported by dopamine, dobutamine, and epinephrine. At day 11, she developed bradycardia, and cardiopulmonary resuscitation was initiated; however, she died.

\section{Discussion}

The SARS-CoV-2 novel virus is known to cause severe pneumonia in adults. The early neonatal infection has been reported frequently, but the reports by Vivanti et al. and Patane et al. are probably the first and only reports confirming vertical transmission $[5,6]$. In our centre, we have confirmed SARS-CoV- 2 infection in 45 pregnant women until the date of this report. Three neonates' PCR test results were positive for the virus: aside from our reported case, another two neonates were asymptomatic. This accounts for a $15 \%$ incidence among infected mothers, compared to 9\% found by Zhu et al. [3], and no cases reported by Schwartz [8], taking the limited sensitivity of nasopharyngeal RT-PCR testing into consideration [9].

In reviewing the presentation of coronavirus among different settings and ages, the clinical picture varies from asymptomatic carriers, severe pneumonia, shock, and thromboembolism to multisystem inflammatory 
syndrome in children (MIS-C). Neonates have been reported to be asymptomatic or to have mild respiratory symptoms or mild pneumonia [4]. One case was reported to have meningoencephalitis [6].

To date, there have been no reported cases of a neonate with severe respiratory symptoms and a severe picture of pneumonia as in our case. The case also exhibited severe persistent pulmonary hypertension that responded poorly to inhaled nitric oxide and respiratory support. The severity of vertical infection of SARS$\mathrm{CoV}-2$ may depend on advancing gestational age, with less infectivity early in pregnancy and a more severe picture, as in our case, associated with late-trimester infection.

Although MIS-C has been highlighted in children infected with the SARS-CoV-2 virus and has been linked to poor prognosis [10], the multiple organ dysfunctions that the neonate in our case study had can be explained by her severe persistent pulmonary hypertension status and hemodynamic instability, especially with lack of skin manifestation, fever, and other signs and symptoms that exist in MIS-C.

In our reported case, the mother was infected and symptomatic shortly before delivery. A caesarean section was performed due to bleeding and abnormal CTG. Our healthcare workers were strictly following the infection-control guidelines related to COVID-19 and followed the pathway for suspected or confirmed COVID-19 cases, which includes the assignment of a trained team from all related subspecialties to attend the delivery, the limitation of healthcare workers to a minimum in a designated isolated operating room, and the use of an $\mathrm{N} 95$ mask by everyone. The operation was held under spinal anaesthesia, and no arousalgenerating procedures were needed. The mother was wearing a surgical mask because she was suspected of having SARS-CoV-2 infection. Resuscitation of the neonate was conducted $3 \mathrm{~m}$ away from the operation site, with full personal protective equipment (PPE). Delayed cord clamping was not applied, and no skin-to-skin contact was allowed between the mother and the newborn. The transferring team was wearing full PPE, and the patient had been in airborne precaution since admission to NICU Level 3. None of the healthcare providers who dealt with the neonate or her mother had symptoms of COVID-19.

The application of all these measures and precautions suggests that the SARS-CoV-2 infection in the neonate was caused by vertical transmission, although droplet transmission cannot be ruled out. Further investigating tools such as sampling the amniotic fluid for the virus, a placenta pathology examination, and a neonate blood sample test for antigens and antibodies would have been required to validate a vertical transmission claim.

Neonatal pneumonia has been reported in babies born to COVID-19 mothers [3,9]. In the Zhu study [3], 6 out of 10 infants born to COVID-19 mothers were experiencing respiratory symptoms. Moreover, seven babies have $\mathrm{x}$-ray findings. This includes pneumonia in four neonates, bilateral blurred lungs and pneumothorax in one neonate, and ground-glass appearance in two other neonates. Although all their RTPCR testing came negative for SARS-CoV-2, small sample size and high rate of false-negative were considered. In our reported case, ground-glass appearance and lung infiltrate may indicate a severe form of a similar pathological picture that lead to the unfortunate outcome. Other differential diagnoses, such as late prematurity with the risk of respiratory distress syndrome or transient tachypnea of the newborn, cannot solely explain the scenario, although it may have worsened the condition and resulted in this severe picture of pneumonia and persistent pulmonary hypertension. Interestingly, angiotensin-converting enzyme 2 (ACE2), which has been recorded as a targeted receptor for SARS-COV-2 [11], plays an important role in the pathogenesis of a variety of lung injuries in a neonate. Furthermore, it has been hypothesized that ACE2 has a role in vascular remodelling and pulmonary hypertension [12]. The claim of whether COVID-19 contributed directly to the pathology of pulmonary hypertension or through lung pneumonia needs to be investigated.

\section{Conclusions}

Owing to the quick deterioration of the neonate, SARS-CoV-2 does not appear to be a benign entity in neonates. It could be very well stated that COVID-19 may be considered as a possible cause of severe pneumonia and subsequently the mortality. Although not the usual presentation, it could have been secondary to a severe course of respiratory distress syndrome of prematurity or other comorbidities, such as primary persistent pulmonary hypertension of the newborn.

\section{Additional Information \\ Disclosures}

Human subjects: Consent was obtained by all participants in this study. Conflicts of interest: In compliance with the ICMJE uniform disclosure form, all authors declare the following: Payment/services info: All authors have declared that no financial support was received from any organization for the submitted work. Financial relationships: All authors have declared that they have no financial relationships at present or within the previous three years with any organizations that might have an interest in the submitted work. Other relationships: All authors have declared that there are no other relationships or activities that could appear to have influenced the submitted work. 


\section{References}

1. COVID-19 coronavirus pandemic. (2020). Accessed: September 15, 2020: https://www.worldometers.info/coronavirus/.

2. Zamaniyan M, Ebadi A, Aghajanpoor Mir S, Rahmani Z, Haghshenas M, Azizi S: Preterm delivery in pregnant woman with critical COVID-19 pneumonia and vertical transmission [Epub ahead of print]. Prenat Diagn. 2020, 10.1002/pd.5713

3. Zhu H, Wang L, Fang C, et al.: Clinical analysis of 10 neonates born to mothers with 2019-nCoV pneumonia Transl Pediatr. 2020, 9:51-60. 10.21037/tp.2020.02.06

4. Zeng L, Xia S, Yuan W, Yan K, Xiao F, Shao J, Zhou W: Neonatal early-onset infection with SARS-CoV-2 in 33 neonates born to mothers with COVID-19 in Wuhan, China. JAMA Pediatr. 2020, 174:722-725. 10.1001/jamapediatrics.2020.0878

5. Patanè L, Morotti D, Giunta MR, et al.: Vertical transmission of coronavirus disease 2019: severe acute respiratory syndrome coronavirus 2 RNA on the fetal side of the placenta in pregnancies with coronavirus disease 2019-positive mothers and neonates at birth. Am J Obstet Gynecol MFM. 2020, 2:100145. 10.1016/j.ajogmf.2020.100145

6. Vivanti AJ, Vauloup-Fellous C, Prevot S, et al.: Transplacental transmission of SARS-CoV-2 infection. Nat Commun. 2020, 14:3572. 10.1038/s41467-020-17436-6

7. Ministry of Health Saudi Arabia. Saudi MoH protocol for patients suspected of/confirmed with COVID-19 . (2020). Accessed: September 15, 2020:

https://www.moh.gov.sa/Ministry/MediaCenter/Publications/Documents/MOH-therapeutic-protocol-forCOVID-19.pdf.

8. Schwartz DA: An analysis of 38 pregnant women with COVID-19, their newborn infants, and maternal-fetal transmission of SARS-CoV- 2: maternal coronavirus infections and pregnancy outcomes. Arch Pathol Lab Med. 2020, 144:799-805. 10.5858/arpa.2020-0901-SA

9. Wang W, Xu Y, Gao R, Lu R, Han K, Wu G, Tan W: Detection of SARS-CoV-2 in different types of clinical specimens. JAMA. 2020, 323:1843-1844. 10.1001/jama.2020.3786

10. Pouletty M, Borocco C, Ouldali N, et al.: Paediatric multisystem inflammatory syndrome temporally associated with SARS-CoV-2 mimicking Kawasaki disease (Kawa-COVID- 19): a multicentre cohort. Ann Rheum Dis. 2020, 79:999-1006. 10.1136/annrheumdis-2020-217960

11. Li W, Moore MJ, Vasilieva N, et al.: Angiotensin-converting enzyme 2 is a functional receptor for the SARS coronavirus. Nature. 2003, 426:450-454. 10.1038/nature02145

12. Gandhi C, Uhal BD: Roles of the angiotensin system in neonatal lung injury and disease . JSM Atheroscler. 2016, 1:1014. 